75

ournal of

Mechanics of

Materials and Structures

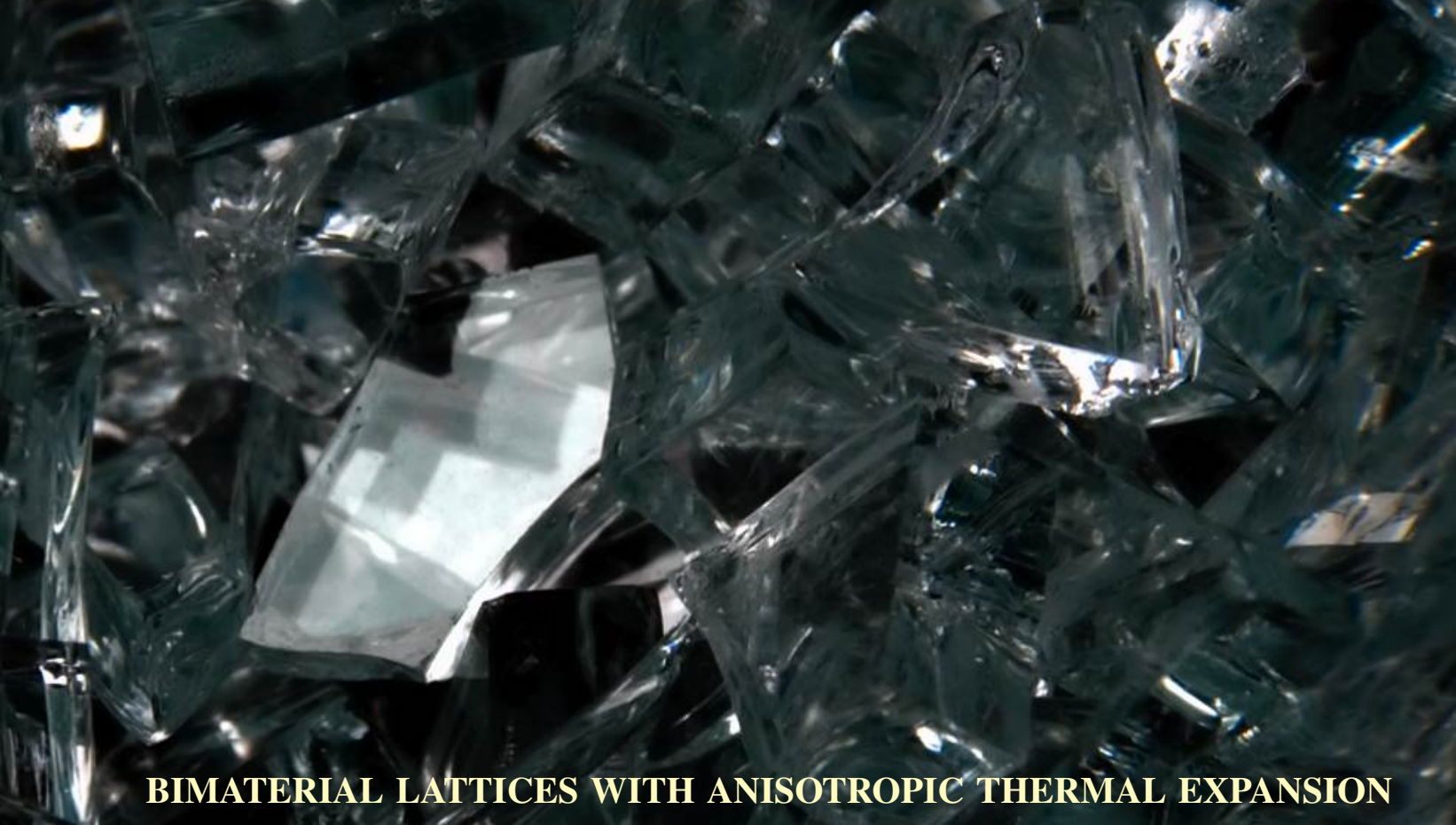

BIMATERIAL LATTICES WITH ANISOTROPIC THERMAL EXPANSION Marina M. Toropova and Craig A. Steeves

2

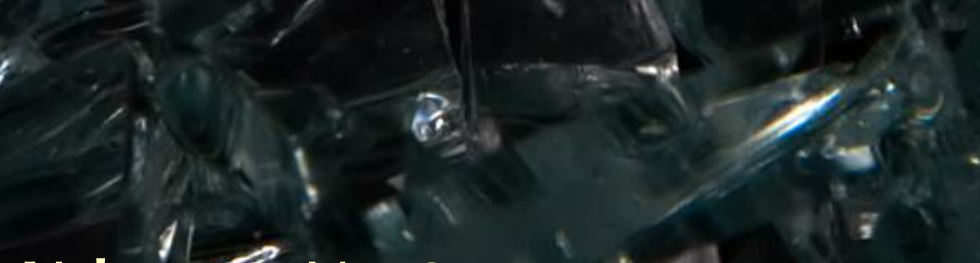

Volume 9 , No. 2

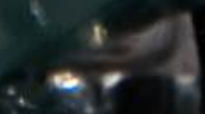

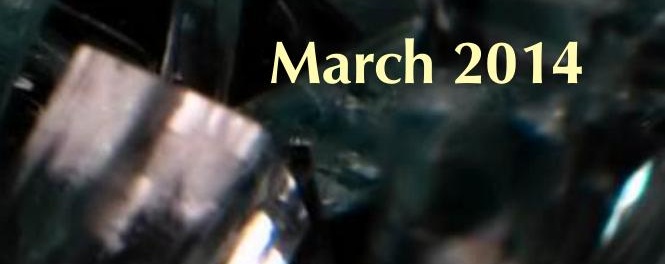




\title{
BIMATERIAL LATTICES WITH ANISOTROPIC THERMAL EXPANSION
}

\author{
Marina M. Toropova And Craig A. Steeves
}

\begin{abstract}
Bimaterial lattices with anisotropic thermal expansion consist of nonidentical cells comprising a skewed triangle made of one material surrounding an unskewed triangle made of a second material. The two materials have differing individual coefficients of thermal expansion. The variation in skew angles makes the thermal expansion of the cell anisotropic. Being composed of anisotropic nonidentical cells, the assembled lattice is also anisotropic and can be designed to have different coefficients of thermal expansion on its top and bottom surfaces. Such lattices can be used as transition elements or adapters to avoid thermal-expansion mismatch between two parts of a structure made of materials with different coefficients of thermal expansion and subjected to temperature changes. In the present paper, three nonlinear algebraic equations linking skew angles with the coefficients of thermal expansion in three directions of a cell are derived, the design of several lattices is performed, the algorithm of lattice design is elaborated, and some examples are presented.
\end{abstract}

\section{Introduction}

Often structures that are used in aerospace, civil engineering, and microelectronics experience large temperature changes. If connected components of such structures are made of materials with different coefficients of thermal expansion (CTE), they experience mechanical stresses due to thermal-expansion mismatch. To eliminate thermal stresses, the parts of the structure with differing CTEs, herein referred to as the substrates, can be connected to each other through special transition elements or adapters with anisotropic CTE. Using composite structures with graded CTEs for joining purposes is not a new concept. For example, Yousefiani et al. [2009a; 2009b] applied it to design a layered injector-chamber attachment component in rocket engines. In the first of these papers they suggested joining approaches such as welding, brazing, or solid-state bonding to produce a graded-CTE layered composite. In the second, they used build-up (bottom-up) fabrication approaches such as metal deposition or powder metallurgy to produce a graded-CTE layered composite preform, which was consolidated and heat-treated to create the graded-CTE integrated composite billet of near net shape. In [Dang 2008], the composite adapters with graded CTE were components of a precision optical assembly to prevent lens misalignment. The adapter material comprised multiple thin composite material layers, each possessing a CTE slightly different from its two adjacent layers, bonded to form an adapter with CTE gradually varying in the direction perpendicular to the bonding interfaces. Such adapters will bend when subjected to temperature changes, and deformations of the system must be permitted or thermal stresses will arise if the bending is suppressed. Also, their mechanical properties can be substantially anisotropic, leading to the reduction of overall stiffness and strength. An alternative to graded systems is a compliant system, where differential

Funding was provided through Natural Sciences and Engineering Research Council Strategic Project Grant 413357-11.

Keywords: composite lattices, graded coefficient of thermal expansion, thermal-mismatch adapters. 
thermal expansion is accommodated by connectors with low stiffness. This has the disadvantage of reducing the overall stiffness of the structure.

Here, we suggest a way for joining dissimilar parts of a structure using planar composite lattices with anisotropic CTE: one edge of the lattice has CTE that matches the material of the first substrate, and the opposite edge of the lattice has a CTE matching the second substrate. In designing such lattices, we rely on [Sigmund and Torquato 1996; Lakes 1996; Gibiansky and Torquato 1997; Sigmund and Torquato 1997; Jefferson et al. 2009; Steeves et al. 2007; Lehman and Lakes 2013], where it was demonstrated that a lattice with a desirable isotropic CTE can be constructed from cells incorporating two materials with widely differing individual CTEs and empty space. A lattice cell consisting of a skewed triangle of low-CTE material surrounding a triangle of high-CTE material combines high stiffness, simple shape, and ability to reach desirable CTE [Steeves et al. 2007]. In [Steeves et al. 2007; Berger et al. 2011], a pinjointed lattice consisting of these cells is shown to be nearly optimally stiff: the mechanical response of this type of lattice is dominated by stretching rather than bending. Such lattices are structurally robust and can be relatively easily manufactured. Gdoutos et al. [2013] designed, fabricated, and tested thermally stable metastructures consisting of the cells suggested in [Steeves et al. 2007]. These structures were finely and coarsely tuned by varying the CTE of the constituent materials and the unit cell geometry. The microscale unit cells were composed of aluminum and titanium and were assembled over a large area to create thin low-CTE foils.

In this work, the lattice concept created by Steeves et al. is used to design a lattice adapter with anisotropic CTE. To be anisotropically tunable, each cell can have six different skew angles and therefore have anisotropic thermal expansion. Also, the cells in the lattice are not identical. As a result, the whole lattice has anisotropic and graded net CTE. In this paper, the anisotropic thermal expansion of the lattice is analyzed and the equations connecting the cell skew angles with CTE in three directions are derived. These equations are used to find desirable skew angles for the design of each cell. Then the design of the whole lattice is performed: three CTEs in each cell are found as functions of the CTEs of the substrates. A system for choosing the lattice materials that can provide such CTEs is discussed. Design examples then show how the choice of materials influences the skewness of the cells. This anisotropic-lattice concept eliminates both of the problems with other adapter concepts: the lattice remains stiff at all times, and if pin-connected, differential thermal deformations of the substrates are accommodated without generating any thermal stresses either in the lattice or the substrates. Moreover, the anisotropic lattices presented here are scale independent and can be extended to three-dimensional geometries.

\section{Formulation of the problem}

Consider two adjoining planar parts of a structure; name them Substrate 1 and Substrate 2. Suppose that Substrates 1 and 2 are made of materials with different CTEs $A_{1}$ and $A_{2}$, respectively. We would like to join these plates by a planar interfacial one-row lattice that has the CTE of the first substrate $A_{1}$ on the edge connected to the first plate and the CTE of the second substrate $A_{2}$ on the edge adjacent to the second plate; this eliminates thermal stresses in the substrates during thermal excursions. In addition, the lattice itself should experience no internal thermal stresses during temperature changes. For this purpose, we use the lattice with cells described in [Steeves et al. 2007]: it is based on a virtual triangle $A C E$ and consists of a skewed triangle $A B C D E F$ made of a material with lower CTE $\alpha_{1}$ and connected with an 


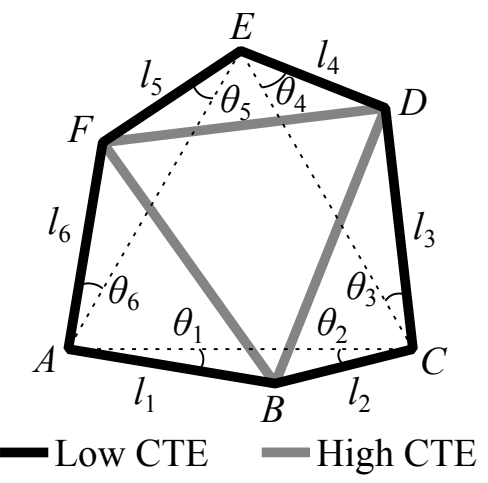

Figure 1. One cell of a lattice. The members $A B, B C, C D, D E, E F$, and $A F$ have low CTE, depicted in black. The members $B D, D F$, and $B F$ have higher CTE, depicted in gray. The equilateral triangle upon which the cell is based is shown as a dashed line. The skew angles, $\theta_{1}, \theta_{2}, \theta_{3}, \theta_{4}, \theta_{5}$, and $\theta_{6}$, are the angles by which the unit cell strut orientation differs from those of an equilateral triangle.

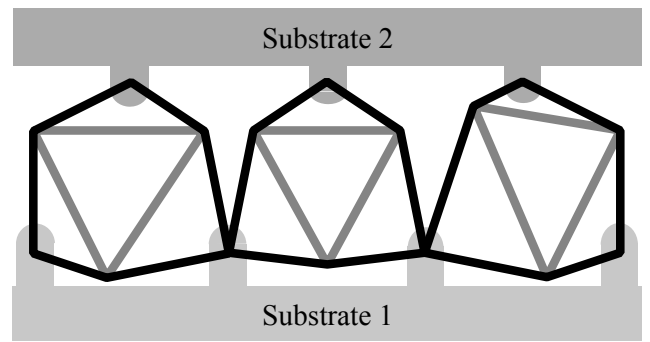

Figure 2. A planar anisotropic lattice connecting two substrates with different CTE. The lattice has net anisotropic CTE, and each individual cell has net anisotropic CTE.

internal unskewed triangle $B D F$ made of a material with higher CTE $\alpha_{2}$ (Figure 1). The internal triangle $B D F$ is pin-joined to the skewed triangle. Adjacent cells of the lattice, connected at $A, C$, and $E$, also have pin-joints between each other and with the substrates (Figure 2) such that the whole structure is free of bending and thermal stresses.

The triangle $A C E$ is equilateral [Steeves et al. 2007]: $A C=L_{1}, C E=L_{2}, A E=L_{3}$, and $L_{1}=$ $L_{2}=L_{3}=L$. Unlike the lattice described in [Steeves et al. 2007], the skew angles $\theta_{1}, \theta_{2}, \theta_{3}, \theta_{4}, \theta_{5}$, and $\theta_{6}$ and the lengths of members $l_{1}=A B, l_{2}=B C, l_{3}=C D, l_{4}=D E, l_{5}=E F$, and $l_{6}=A F$ may be different. As a result, $A C, C E$, and $A E$ can expand differently when temperature changes: $d L_{1}=\overline{\alpha_{1}} L_{1} d T, d L_{2}=\overline{\alpha_{2}} L_{2} d T$, and $d L_{3}=\overline{\alpha_{3}} L_{3} d T$, where $T$ is temperature and $\overline{\alpha_{i}}, i=1,2,3$, are the CTEs of the cell along $A C, C E$, and $A E$, respectively.

By choosing the angles $\theta_{i}, i=1,2,3,4,5,6$, we can influence the change of $d L_{1}, d L_{2}$, and $d L_{3}$ in each cell and provide different changes of distances between vertices of the cells and hence different CTEs on the bottom and top levels of the lattice (Figure 3). To design such a lattice, we need first to obtain relations between CTEs $\overline{\alpha_{i}}, i=1,2,3$, and angles $\theta_{j}, j=1,2,3,4,5,6$. 




Figure 3. Lattice design: base equilateral triangles of two cells. During thermal expansion, the original points translate from, for example, $B$ to $B^{\prime}$. The lattice cell must be designed to accommodate the changing distances between the points.

\section{General formulae}

The undeformed lengths of the constituent members of a cell (Figure 1) are

$$
\begin{array}{lll}
l_{1}=L_{1} f_{1}\left(\theta_{1}, \theta_{2}\right), & l_{2}=L_{1} f_{2}\left(\theta_{1}, \theta_{2}\right), & l_{3}=L_{2} f_{3}\left(\theta_{3}, \theta_{4}\right), \\
l_{4}=L_{2} f_{4}\left(\theta_{3}, \theta_{4}\right), & l_{5}=L_{3} f_{5}\left(\theta_{5}, \theta_{6}\right), & l_{6}=L_{3} f_{6}\left(\theta_{5}, \theta_{6}\right),
\end{array}
$$

where

$$
\begin{array}{lll}
f_{1}=\frac{\sin \theta_{2}}{\sin \left(\theta_{1}+\theta_{2}\right)}, & f_{2}=\frac{\sin \theta_{1}}{\sin \left(\theta_{1}+\theta_{2}\right)}, & f_{3}=\frac{\sin \theta_{4}}{\sin \left(\theta_{3}+\theta_{4}\right)}, \\
f_{4}=\frac{\sin \theta_{3}}{\sin \left(\theta_{3}+\theta_{4}\right)}, & f_{5}=\frac{\sin \theta_{6}}{\sin \left(\theta_{5}+\theta_{6}\right)}, & f_{6}=\frac{\sin \theta_{5}}{\sin \left(\theta_{5}+\theta_{6}\right)} .
\end{array}
$$

From (1), we obtain the differential relations

$$
\begin{aligned}
& \frac{1}{L} d l_{1}\left(1-\frac{\overline{\alpha_{1}}}{\alpha_{1}}\right)=f_{1_{\theta_{1}}}^{\prime} d \theta_{1}+f_{1_{\theta_{2}}}^{\prime} d \theta_{2}, \quad \frac{1}{L} d l_{2}\left(1-\frac{\overline{\alpha_{1}}}{\alpha_{1}}\right)=f_{2_{\theta_{1}}}^{\prime} d \theta_{1}+f_{2_{\theta_{2}}}^{\prime} d \theta_{2}, \\
& \frac{1}{L} d l_{3}\left(1-\frac{\overline{\alpha_{2}}}{\alpha_{1}}\right)=f_{3_{\theta_{3}}}^{\prime} d \theta_{3}+f_{3_{\theta_{4}}}^{\prime} d \theta_{4}, \quad \frac{1}{L} d l_{4}\left(1-\frac{\overline{\alpha_{2}}}{\alpha_{1}}\right)=f_{4_{\theta_{3}}}^{\prime} d \theta_{3}+f_{4_{\theta_{4}}}^{\prime} d \theta_{4}, \\
& \frac{1}{L} d l_{5}\left(1-\frac{\overline{\alpha_{3}}}{\alpha_{1}}\right)=f_{5_{\theta_{5}}}^{\prime} d \theta_{5}+f_{5_{\theta_{6}}}^{\prime} d \theta_{6}, \quad \frac{1}{L} d l_{6}\left(1-\frac{\overline{\alpha_{3}}}{\alpha_{1}}\right)=f_{6_{\theta_{5}}}^{\prime} d \theta_{5}+f_{6_{\theta_{6}}}^{\prime} d \theta_{6},
\end{aligned}
$$

where $f_{i_{\theta_{j}}}^{\prime}, i, j=1,2,3,4,5,6$, is a partial derivative of the function $f_{i}$ with respect to the angle $\theta_{j}$.

The length of three members of the internal triangle can be expressed as

$$
\begin{aligned}
& F B=l_{7}=\left(L_{1}^{2} f_{1}^{2}+L_{3}^{2} f_{6}^{2}-2 L_{1} L_{3} f_{1} f_{6} \cos A^{\prime}\right)^{1 / 2}, \\
& B D=l_{8}=\left(L_{1}^{2} f_{2}^{2}+L_{2}^{2} f_{3}^{2}-2 L_{1} L_{2} f_{2} f_{3} \cos B^{\prime}\right)^{1 / 2}, \\
& D F=l_{9}=\left(L_{2}^{2} f_{4}^{2}+L_{3}^{2} f_{5}^{2}-2 L_{2} L_{3} f_{4} f_{5} \cos C^{\prime}\right)^{1 / 2},
\end{aligned}
$$

where

$$
A^{\prime}=\theta_{1}+\theta_{6}+60^{\circ}, \quad B^{\prime}=\theta_{2}+\theta_{3}+60^{\circ}, \quad \text { and } \quad C^{\prime}=\theta_{4}+\theta_{5}+60^{\circ} .
$$


From (3), we can find $d l_{i}, i=7,8,9$, as

$$
\begin{aligned}
& d l_{7}=a_{7} d l_{7}+b_{7} d \theta_{1}+c_{7} d \theta_{2}+d_{7} d \theta_{5}+e_{7} d \theta_{6}, \\
& d l_{8}=a_{8} d l_{8}+b_{8} d \theta_{1}+c_{8} d \theta_{2}+d_{8} d \theta_{3}+e_{8} d \theta_{4}, \\
& d l_{9}=a_{9} d l_{9}+b_{9} d \theta_{3}+c_{9} d \theta_{4}+d_{9} d \theta_{5}+e_{9} d \theta_{6},
\end{aligned}
$$

where

$$
\begin{aligned}
& a_{7}=\frac{l_{1}}{l_{7}^{2}}\left(l_{1}-l_{6} \cos A^{\prime}\right) \frac{\overline{\alpha_{1}}}{\alpha_{2}}+\frac{l_{6}}{l_{7}^{2}}\left(l_{6}-l_{1} \cos A^{\prime}\right) \frac{\overline{\alpha_{3}}}{\alpha_{2}}, \\
& b_{7}=\frac{\partial l_{7}}{\partial \theta_{1}}=\frac{L}{l_{7}}\left(l_{1}-l_{6} \cos A^{\prime}\right) f_{1_{\theta_{1}}}^{\prime}+\frac{l_{1} l_{6}}{l_{7}} \sin A^{\prime}, \\
& c_{7}=\frac{\partial l_{7}}{\partial \theta_{2}}=\frac{L}{l_{7}}\left(l_{1}-l_{6} \cos A^{\prime}\right) f_{1_{\theta_{2}}}^{\prime}, \\
& d_{7}=\frac{\partial l_{7}}{\partial \theta_{5}}=\frac{L}{l_{7}}\left(l_{6}-l_{1} \cos A^{\prime}\right) f_{6_{\theta_{5}}}^{\prime}, \\
& e_{7}=\frac{\partial l_{7}}{\partial \theta_{6}}=\frac{L}{l_{7}}\left(l_{6}-l_{1} \cos A^{\prime}\right) f_{6_{\theta_{6}}}^{\prime}+\frac{l_{1} l_{6}}{l_{7}} \sin A^{\prime} .
\end{aligned}
$$

The coefficients $a_{8}, b_{8}, c_{8}, d_{8}$, and $e_{8}$ can be obtained from previous formulae by replacing $l_{1}, l_{6}, l_{7}, \overline{\alpha_{1}}$, $\overline{\alpha_{3}}, f_{1}, f_{6}, \theta_{1}, \theta_{2}, \theta_{5}, \theta_{6}$, and $A^{\prime}$ by $l_{3}, l_{2}, l_{8}, \overline{\alpha_{2}}, \overline{\alpha_{1}}, f_{3}, f_{2}, \theta_{3}, \theta_{4}, \theta_{1}, \theta_{2}$, and $B^{\prime}$, respectively. Similarly, the coefficients $a_{9}, b_{9}, c_{9}, d_{9}$, and $e_{9}$ can be obtained from (5) by replacing $l_{1}, l_{6}, l_{7}, \overline{\alpha_{1}}, \overline{\alpha_{3}}, f_{1}, f_{6}, \theta_{1}$, $\theta_{2}, \theta_{5}, \theta_{6}$, and $A^{\prime}$ by $l_{5}, l_{4}, l_{9}, \overline{\alpha_{3}}, \overline{\alpha_{2}}, f_{5}, f_{4}, \theta_{5}, \theta_{6}, \theta_{3}, \theta_{4}$, and $C^{\prime}$, respectively.

Then expressing $d \theta_{i}$ through $d l_{i}$ from (2), substituting them into (4), and performing all necessary transformations, we obtain three nonlinear equations linking the six skew angles $\theta_{i}$ with three normalized thermal-expansion coefficients along the lines $A C, C E$, and $A E$ in the skewed triangle:

$$
\begin{gathered}
\left(\frac{\overline{\alpha_{1}}}{\alpha_{1}}-1\right) \frac{f_{1} \cos \left(\theta_{1}+\theta_{2}\right)+f_{2}}{\sin \theta_{2}}+\left(\frac{\overline{\alpha_{3}}}{\alpha_{1}}-1\right) \frac{f_{6} \cos \left(\theta_{5}+\theta_{6}\right)+f_{5}}{\sin \theta_{5}}=\frac{1}{\sin A^{\prime}}\left(1-\frac{\alpha_{2}}{\alpha_{1}}\right)\left(\frac{f_{1}}{f_{6}}+\frac{f_{6}}{f_{1}}-2 \cos A^{\prime}\right), \\
\left(\frac{\overline{\alpha_{1}}}{\alpha_{1}}-1\right) \frac{f_{2} \cos \left(\theta_{1}+\theta_{2}\right)+f_{1}}{\sin \theta_{1}}+\left(\frac{\overline{\alpha_{2}}}{\alpha_{1}}-1\right) \frac{f_{3} \cos \left(\theta_{3}+\theta_{4}\right)+f_{4}}{\sin \theta_{4}}=\frac{1}{\sin B^{\prime}}\left(1-\frac{\alpha_{2}}{\alpha_{1}}\right)\left(\frac{f_{2}}{f_{3}}+\frac{f_{3}}{f_{2}}-2 \cos B^{\prime}\right), \\
\left(\frac{\overline{\alpha_{3}}}{\alpha_{1}}-1\right) \frac{f_{5} \cos \left(\theta_{5}+\theta_{6}\right)+f_{6}}{\sin \theta_{6}}+\left(\frac{\overline{\alpha_{2}}}{\alpha_{1}}-1\right) \frac{f_{4} \cos \left(\theta_{3}+\theta_{4}\right)+f_{3}}{\sin \theta_{3}}=\frac{1}{\sin C^{\prime}}\left(1-\frac{\alpha_{2}}{\alpha_{1}}\right)\left(\frac{f_{4}}{f_{5}}+\frac{f_{5}}{f_{4}}-2 \cos C^{\prime}\right) .
\end{gathered}
$$

The equations (6) are scale-independent, contain the ratio $\alpha_{2} / \alpha_{1}$ as a parameter, and couple three normalized CTEs in a cell $\overline{\alpha_{1}} / \alpha_{1}, \overline{\alpha_{2}} / \alpha_{1}$, and $\overline{\alpha_{3}} / \alpha_{1}$. If the skew angles are known, these three CTEs 
can be calculated using the following formulae:

$$
\frac{\overline{\alpha_{1}}}{\alpha_{1}}=1+\frac{\Delta_{1}}{\Delta}, \quad \frac{\overline{\alpha_{2}}}{\alpha_{1}}=1+\frac{\Delta_{2}}{\Delta}, \quad \text { and } \quad \frac{\overline{\alpha_{3}}}{\alpha_{1}}=1+\frac{\Delta_{3}}{\Delta},
$$

where

$$
\begin{aligned}
\Delta & =c_{11} c_{22} c_{33}+c_{21} c_{32} c_{13}, \\
\Delta_{1} & =F_{1} c_{22} c_{33}+F_{2} c_{13} c_{32}-F_{3} c_{22} c_{13}, \\
\Delta_{2} & =F_{2} c_{11} c_{33}+F_{3} c_{13} c_{21}-F_{1} c_{21} c_{33}, \\
\Delta_{3} & =F_{3} c_{11} c_{22}+F_{1} c_{21} c_{32}-F_{2} c_{11} c_{32},
\end{aligned}
$$

$$
\begin{array}{r}
F_{1}=\frac{1}{\sin A^{\prime}}\left(1-\frac{\alpha_{2}}{\alpha_{1}}\right)\left(\sin ^{2} \theta_{2} \sin ^{2}\left(\theta_{5}+\theta_{6}\right)+\sin ^{2} \theta_{5} \sin ^{2}\left(\theta_{1}+\theta_{2}\right)\right. \\
\left.\quad-2 \cos A^{\prime} \sin \theta_{2} \sin \theta_{5} \sin \left(\theta_{1}+\theta_{2}\right) \sin \left(\theta_{5}+\theta_{6}\right)\right), \\
\begin{array}{r}
F_{2}=\frac{1}{\sin B^{\prime}}\left(1-\frac{\alpha_{2}}{\alpha_{1}}\right)\left(\sin ^{2} \theta_{1} \sin ^{2}\left(\theta_{3}+\theta_{4}\right)+\sin ^{2} \theta_{4} \sin ^{2}\left(\theta_{1}+\theta_{2}\right)\right. \\
\left.\quad-2 \cos B^{\prime} \sin \theta_{1} \sin \theta_{4} \sin \left(\theta_{1}+\theta_{2}\right) \sin \left(\theta_{3}+\theta_{4}\right)\right), \\
F_{3}=\frac{1}{\sin C^{\prime}}\left(1-\frac{\alpha_{2}}{\alpha_{1}}\right)\left(\sin ^{2} \theta_{3} \sin ^{2}\left(\theta_{5}+\theta_{6}\right)+\sin ^{2} \theta_{6} \sin ^{2}\left(\theta_{3}+\theta_{4}\right)\right. \\
\left.\quad-2 \cos C^{\prime} \sin \theta_{3} \sin \theta_{6} \sin \left(\theta_{3}+\theta_{4}\right) \sin \left(\theta_{5}+\theta_{6}\right)\right) .
\end{array}
\end{array}
$$

On the other hand, in design of anisotropic lattices, we need to solve the inverse problem of finding the skew angles when the values of three CTEs in each cell are known. In this case, three equations (6) are insufficient and it is possible to impose additional conditions on the lattice or optimize the lattice structure using various criteria. If instead we make the simplification $\theta_{1}=\theta_{2}=t_{1}, \theta_{3}=\theta_{4}=t_{2}$, and $\theta_{5}=\theta_{6}=t_{3}$ (Figure 4), the equations (6) take the form

$$
\begin{aligned}
& \left(\frac{\overline{\alpha_{1}}}{\alpha_{1}}-1\right) \cot t_{1}+\left(\frac{\overline{\alpha_{3}}}{\alpha_{1}}-1\right) \cot t_{3}=G_{1}, \\
& \left(\frac{\overline{\alpha_{1}}}{\alpha_{1}}-1\right) \cot t_{1}+\left(\frac{\overline{\alpha_{2}}}{\alpha_{1}}-1\right) \cot t_{2}=G_{2}, \\
& \left(\frac{\overline{\alpha_{2}}}{\alpha_{1}}-1\right) \cot t_{2}+\left(\frac{\overline{\alpha_{3}}}{\alpha_{1}}-1\right) \cot t_{3}=G_{3},
\end{aligned}
$$




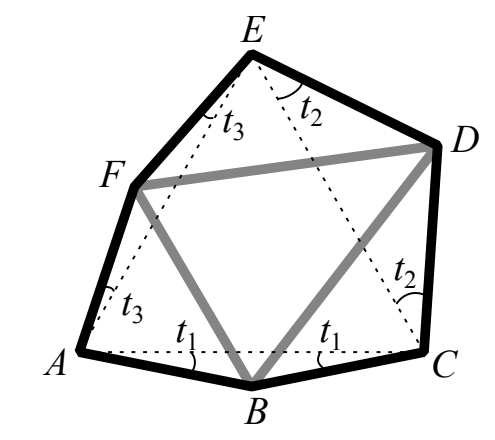

- Low CTE High CTE

Figure 4. A cell with three skew angles. The original six skew angles are replaced by $\theta_{1}=\theta_{2}=t_{1}, \theta_{3}=\theta_{4}=t_{2}$, and $\theta_{5}=\theta_{6}=t_{3}$.

where

$$
\begin{aligned}
G_{1} & =\frac{1}{\sin A^{\prime \prime}}\left(1-\frac{\alpha_{2}}{\alpha_{1}}\right)\left(\frac{\cos t_{1}}{\cos t_{3}}+\frac{\cos t_{3}}{\cos t_{1}}-2 \cos A^{\prime \prime}\right), \\
G_{2} & =\frac{1}{\sin B^{\prime \prime}}\left(1-\frac{\alpha_{2}}{\alpha_{1}}\right)\left(\frac{\cos t_{1}}{\cos t_{2}}+\frac{\cos t_{2}}{\cos t_{1}}-2 \cos B^{\prime \prime}\right), \\
G_{3} & =\frac{1}{\sin C^{\prime \prime}}\left(1-\frac{\alpha_{2}}{\alpha_{1}}\right)\left(\frac{\cos t_{2}}{\cos t_{3}}+\frac{\cos t_{3}}{\cos t_{2}}-2 \cos C^{\prime \prime}\right)
\end{aligned}
$$

and $A^{\prime \prime}=t_{1}+t_{3}+60^{\circ}, B^{\prime \prime}=t_{1}+t_{2}+60^{\circ}$, and $C^{\prime \prime}=t_{2}+t_{3}+60^{\circ}$.

From (11), $\overline{\alpha_{i}} / \alpha_{1}, i=1,2,3$, can be found as

$$
\begin{aligned}
& \frac{\overline{\alpha_{1}}}{\alpha_{1}}=1+\frac{G_{1}+G_{2}-G_{3}}{2} \tan t_{1}, \\
& \frac{\overline{\alpha_{2}}}{\alpha_{1}}=1+\frac{G_{2}+G_{3}-G_{1}}{2} \tan t_{2}, \\
& \frac{\overline{\alpha_{3}}}{\alpha_{1}}=1+\frac{G_{1}+G_{3}-G_{2}}{2} \tan t_{3} .
\end{aligned}
$$

From (12), it is seen that if any $t_{i}=0, i=1,2,3$, then $\overline{\alpha_{i}}=\alpha_{1}$ and the two other skew angles do not influence it. Conversely, if $\overline{\alpha_{i}}=\alpha_{1}$, then the two skew angles adjacent to this side must be equal to zero.

If all angles in (6) are equal to $\theta$, the cell is isotropic with a constant coefficient of thermal expansion in all directions equal to $\bar{\alpha}$ and these three equations can be transformed into the equation for the expansion coefficient obtained in [Steeves et al. 2007]:

$$
\frac{\bar{\alpha}}{\alpha_{1}}=\frac{1-0.5\left(\alpha_{2} / \alpha_{1}\right) \sin (2 \theta)(1 / \sqrt{3}+\tan \theta)}{1-0.5 \sin (2 \theta)(1 / \sqrt{3}+\tan \theta)} .
$$

In the isotropic configuration, the maximum of the function $\bar{\alpha} / \alpha_{1}$ is reached at $\theta=-15^{\circ}$; the function decreases at $-15^{\circ}<\theta<90^{\circ}$ (see Figure 5). For design considerations, to avoid overlapping the cells, we consider skew angles in the range $\left[-15^{\circ}, 30^{\circ}\right]$. In this range of skew angle, all values of three normalized CTEs presented by the formulae (12) belong to the interval $\Omega \equiv\left[\bar{\alpha}\left(30^{\circ}\right) / \alpha_{1}, \bar{\alpha}\left(-15^{\circ}\right) / \alpha_{1}\right]$ that depends 


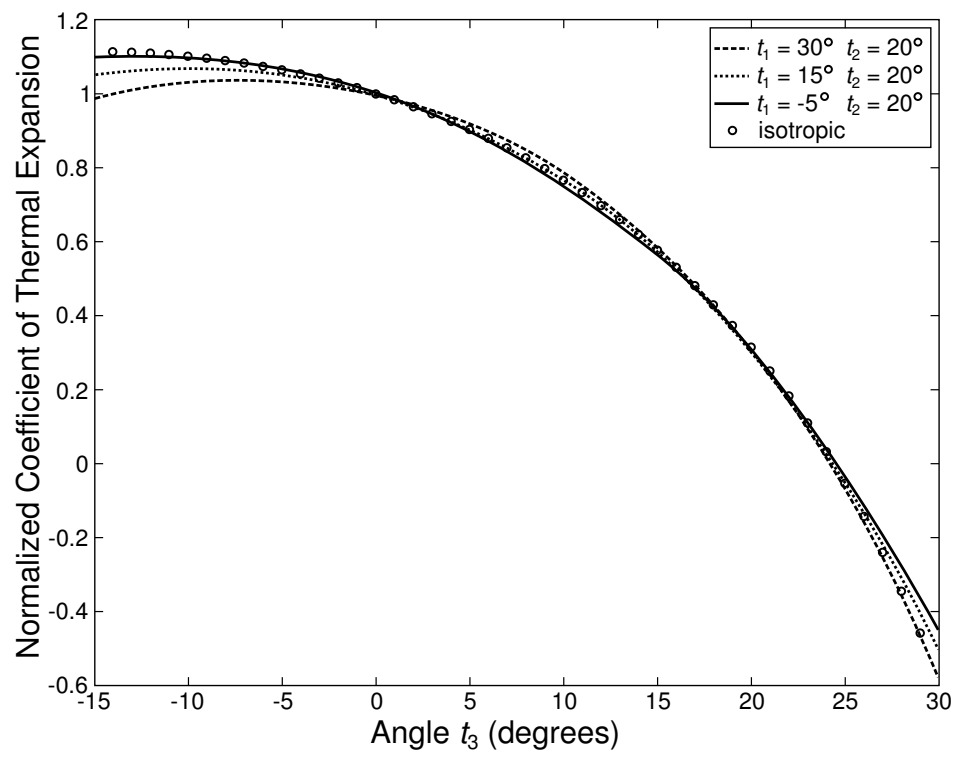

Figure 5. The influence of two skew angles on $\overline{\alpha_{3}} / \alpha_{1}$ for $\alpha_{2} / \alpha_{1}=2.581$, which corresponds to the ratio of the CTEs of aluminum and titanium.

on the ratio $\alpha_{2} / \alpha_{1}$ (Figure 6). Here, $\bar{\alpha} / \alpha_{1}$ is calculated from (13). Figure 5 plots the behavior of $\overline{\alpha_{3}} / \alpha_{1}$ via $t_{3}$ at three different sets of values $t_{1}=30^{\circ}, t_{2}=20^{\circ} ; t_{1}=15^{\circ}, t_{2}=20^{\circ}$; and $t_{1}=-5^{\circ}, t_{2}=20^{\circ}$ and the ratio $\alpha_{2} / \alpha_{1}=2.581$, which corresponds to the ratio of the CTEs of aluminum and titanium. These do not coincide with the isotropic case, which means that an arbitrary set of three values from $\Omega$ is not necessarily a solution of the equations (11). In other words, the condition

$$
\frac{\overline{\alpha_{1}}}{\alpha_{1}}, \frac{\overline{\alpha_{2}}}{\alpha_{1}}, \frac{\overline{\alpha_{3}}}{\overline{\alpha_{1}}} \in \Omega
$$

is necessary but not sufficient for the existence of the solution of the equations (11). For example, while the maximum for $t_{1}=-5^{\circ}, t_{2}=20^{\circ}$ coincides with the maximum for the isotropic case, $t_{1}=15^{\circ}, t_{2}=20^{\circ}$ has a maximum at $t_{3}=-10^{\circ}$ and $t_{1}=30^{\circ}, t_{2}=20^{\circ}$ has a maximum at $t_{3}=-5^{\circ}$. Hence, in the latter two cases, we need to search for solutions in the interval $\left[-10^{\circ}, 30^{\circ}\right]$ and $\left[-5^{\circ}, 30^{\circ}\right]$, respectively. Also, the maxima in the three cases are different, so it is not possible to attain the same maximum $\overline{\alpha_{3}}$.

In Figure 6, it is seen that the interval $\Omega$ increases with respect to the ratio $\alpha_{2} / \alpha_{1}$. The higher values of normalized CTEs can be reached at negative unequal skew angles $\theta_{1} \neq \theta_{2}, \theta_{3} \neq \theta_{4}$, and $\theta_{5} \neq \theta_{6}$. This case will be illustrated in Section 6, Problem 5.

\section{Lattice design}

In this section, planar, one-row lattices are considered. We can design lattices by designating the points on the substrates to which the lattice will be attached and following those points as the temperature changes. This will provide the changes in the lengths of the sides of the equilateral triangles upon which the lattice cells are based, to which the changing lengths of the lattice cells must be matched. For example, Figure 3 shows the base triangles of two cells. Suppose the point $A$ is fixed. When temperature changes, the other 


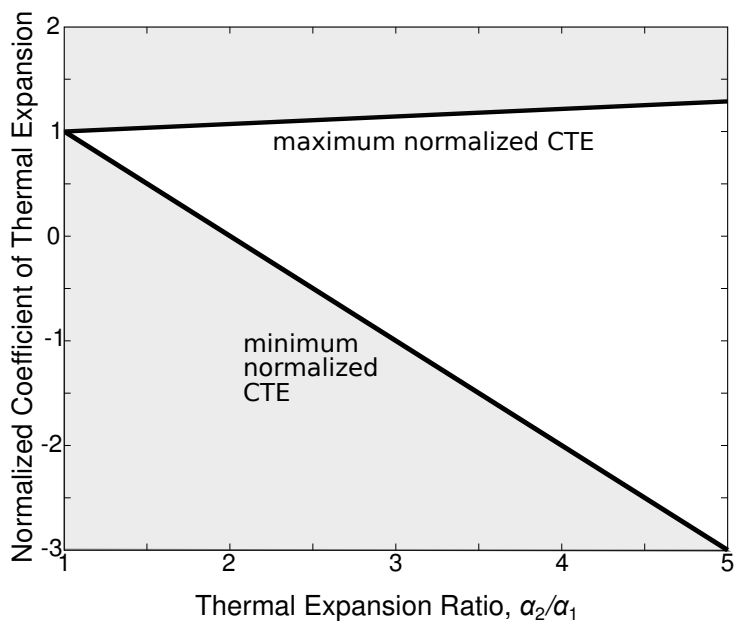

Figure 6. Upper and lower boundaries of the normalized CTE, giving the range $\Omega$, as a function of the ratio $\alpha_{2} / \alpha_{1}$.

points $B, C, D$, and $E$ move to the positions $B^{\prime}, C^{\prime}, D^{\prime}$, and $E^{\prime}$, respectively. The new distances $A B^{\prime}$, $B^{\prime} D^{\prime}$, and $C^{\prime} E^{\prime}$ can be expressed in terms of the side length $L$ and three unknown CTEs of each cell. The following four conditions on the shape of the lattice after temperature changes are sufficient to find them:

(1) the distances between vertices connected to Substrate 1 must be equal to $L\left(1+A_{1} d T\right)$,

(2) the distances between vertices connected to Substrate 2 must be equal to $L\left(1+A_{2} d T\right)$,

(3) the height of each triangle must remain constant, i.e., not depend on temperature, and

(4) the lattice must have a line of symmetry.

The third condition is arbitrary: the relative approach or retreat of the substrates depends upon the materials and the configuration of the overall structure. Here, constant $H$ is chosen although $\Delta H>0$ and $\Delta H<0$ may equivalently be selected.

Using these conditions and deriving formulae for $\overline{\alpha_{i}}, i=1,2,3$, we neglect terms with $\bar{\alpha}_{i}^{2}$ (which is reasonable because $\overline{\alpha_{i}}$ are small). As a result, the formulae for $\overline{\alpha_{1}}, \overline{\alpha_{2}}$, and $\overline{\alpha_{3}}$ in each cell of two-cell, three-cell, and $n$-cell lattices as functions of the CTEs of substrates $A_{1}$ and $A_{2}$ are obtained.

To work with several cells, denote $\alpha_{i 1}=\overline{\alpha_{1}}, \alpha_{i 2}=\overline{\alpha_{2}}$, and $\alpha_{i 3}=\overline{\alpha_{3}}$, where $i$ is the number of the cell in the row (the skeleton of a lattice is depicted in Figure 7). For example, $i=1$ for cell $A B C$ and $i=2$ for $B D E$. Along $A B$ and $B D, j=1$; along $B C$ and $D E, j=2$; and along $A C$ and $B E, j=3$.

Consider a lattice consisting of two cells $A B C$ and $B D E$ (Figure 7). The lattice is pin-joined at $A, B$, and $D$ to a substrate with CTE $A_{1}$ while at points $C$ and $E$ it is pin-joined to a substrate with CTE $A_{2}$. At the initial state, $A B=B C=A C=B D=D E=B E=L$. When the temperature changes, the lengths of these segments become $L\left(1+\alpha_{i j} d T\right)$. The first substrate and the bottom level of the lattice have the same CTE (Condition (1)), $\alpha_{11}=\alpha_{21}=A_{1}$. Similarly, when temperature changes, the distance between points $C$ and $E$ changes following the formula $L\left(1+A_{2} d T\right.$ ) (Condition (2)). Denote $F B=x$. Then for the first cell,

$$
L^{2}\left(1+\alpha_{12} d T\right)^{2}-x^{2}=L^{2}\left(1+\alpha_{13} d T\right)^{2}-\left(L\left(1+\alpha_{11} d T\right)-x\right)^{2} .
$$




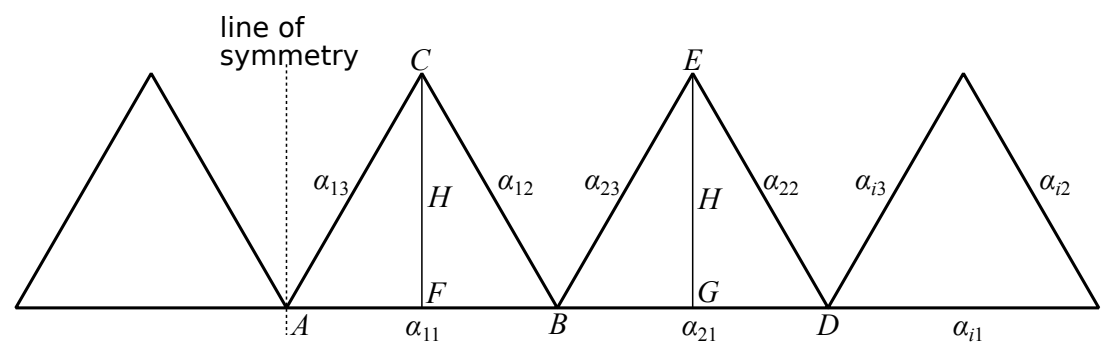

Figure 7. Designation of CTEs in a multicell lattice. Note that the lines do not represent actual cell members; these lines refer to the virtual equilateral triangles upon which the cells are based, shown in Figure 1 as dashed lines. The cell vertices are $A$ to $E$ while the midpoints on the lower face of the cell are $F$ and $G . H$ is the height of the cells. If the lattice has a line of symmetry, it would exist as the dashed line with additional cells to the left.

Neglecting terms with $\alpha_{i j}^{2}$, we get

$$
x=0.5 L\left(1+\left(\alpha_{11}+2 \alpha_{12}-2 \alpha_{13}\right) d T\right) .
$$

Using this formula, we can find from the second cell that

$$
B G=0.5 L\left(1+\left(\alpha_{21}-2 \alpha_{22}+2 \alpha_{23}\right) d T\right) .
$$

Thus, when temperature changes, the distance between points $C$ and $E$ changes according to the rule

$$
C E=L\left(1+\left(\alpha_{12}-\alpha_{13}-\alpha_{22}+\alpha_{23}+A_{1}\right) d T\right) .
$$

Hence,

$$
A_{2}=\alpha_{12}-\alpha_{13}-\alpha_{22}+\alpha_{23}+A_{1}
$$

Also, we would like to design a lattice that does not expand along $F C$ and $G E$ so that the lattice cell height $H$ remains constant when temperature changes (Condition (3)). Applying the approach used above, we can write, for example, for the first cell

$$
\begin{aligned}
(H+d H)^{2}=L^{2}\left(1+\alpha_{12} d T\right)^{2}-0.25 L^{2}\left(1+\left(2 \alpha_{12}-2 \alpha_{13}+\alpha_{11}\right) d T\right)^{2} & \\
& =\left(0.5 \sqrt{3} L\left(1+\frac{2}{3}\left(\alpha_{12}+\alpha_{13}-0.5 \alpha_{11}\right) d T\right)\right)^{2}
\end{aligned}
$$

Thus, the CTE along cell heights $F C$ and $G E$ is $\alpha_{H}=\frac{2}{3}\left(\alpha_{12}+\alpha_{13}-0.5 \alpha_{11}\right)$. In this case,

$$
\alpha_{12}+\alpha_{13}-0.5 \alpha_{11}=0
$$

Finally, for more uniform deformation of the lattice, symmetry can be imposed through $\alpha_{12}=\alpha_{23}$ and $\alpha_{13}=\alpha_{22}$ (Condition (4)). 
For a lattice consisting of two symmetric cells, three CTEs in each cell can be found as functions of the CTEs of the substrates

$$
\begin{aligned}
& \alpha_{11}=\alpha_{21}=A_{1}, \\
& \alpha_{12}=\alpha_{23}=0.25 A_{2}, \\
& \alpha_{13}=\alpha_{22}=0.5 A_{1}-0.25 A_{2} .
\end{aligned}
$$

All values must belong to the interval $\Omega$, and the CTEs, $\alpha_{1}$ and $\alpha_{2}$, of the two materials that comprise the lattice are restricted by the following inequalities:

$$
\begin{aligned}
\alpha_{2}+A_{1} & >2 \alpha_{1}, \\
\alpha_{2}+0.25 A_{2} & >2 \alpha_{1}, \\
\alpha_{2}+0.5 A_{1}-0.25 A_{2} & >2 \alpha_{1}, \\
\alpha_{2}+12.93 \alpha_{1} & >13.93 A_{1}, \\
\alpha_{2}+12.93 \alpha_{1} & >3.48 A_{2}, \\
\alpha_{2}+12.93 \alpha_{1} & >6.97 A_{1}-3.48 A_{2} .
\end{aligned}
$$

From (15), it follows that, in the particular case when the lattice is made of the same materials as the substrates $\left(\alpha_{1}=A_{1}\right.$ and $\left.\alpha_{2}=A_{2}\right)$, the ratio of the lattice material CTEs must lie in the range $2<\alpha_{2} / \alpha_{1}<5.20875$.

For a three-cell lattice, we obtain similar formulae:

$$
\begin{aligned}
& \alpha_{11}=\alpha_{21}=\alpha_{31}=A_{1}, \\
& \alpha_{12}=\alpha_{33}=0.5 A_{2}-0.25 A_{1}, \\
& \alpha_{13}=\alpha_{32}=0.75 A_{1}-0.5 A_{2}, \\
& \alpha_{22}=\alpha_{23}=0.25 A_{1} .
\end{aligned}
$$

The conditions on the CTEs of the materials comprising the lattice are:

$$
\begin{aligned}
\alpha_{2}+0.25 A_{1} & >2 \alpha_{1}, \\
\alpha_{2}+0.5 A_{2}-0.25 A_{1} & >2 \alpha_{1}, \\
\alpha_{2}+0.75 A_{1}-0.5 A_{2} & >2 \alpha_{1}, \\
\alpha_{2}+12.93 \alpha_{1} & >13.93 A_{1}, \\
\alpha_{2}+12.93 \alpha_{1} & >6.97 A_{2}-3.48 A_{1}, \\
\alpha_{2}+12.93 \alpha_{1} & >10.45 A_{1}-6.97 A_{2} .
\end{aligned}
$$

From (17), it follows that, if $\alpha_{1}=A_{1}$ and $\alpha_{2}=A_{2}, 2.5<\alpha_{2} / \alpha_{1}<2.7515$. This is a very narrow range with few candidate materials, so for the lattices consisting of three or more cells, choosing lattice materials different from the substrate materials is nearly obligatory. Aluminum and titanium are a rare combination of common substrate materials that can be connected by a three-cell lattice of the same materials. 
If the lattice contains $2 n$ cells, $n=2,3,4, \ldots$, and is symmetrical with respect to the vertical line passing through one of the bottom vertices (see the line of symmetry in Figure 7), the formulae for cells lying to the right of the symmetry line are

$$
\begin{aligned}
& \alpha_{i 1}=A_{1}, \\
& \alpha_{i 2}=0.5 i A_{1}-0.25(2 i-1) A_{2}, \\
& \alpha_{i 3}=0.25(2 i-1) A_{2}-0.5(i-1) A_{1},
\end{aligned}
$$

where $i=1,2, \ldots, n$.

The conditions for the lattice materials are

$$
\begin{aligned}
\alpha_{2}+A_{1} & >2 \alpha_{1}, \\
\alpha_{2}-0.25 A_{2}+0.5 A_{1}+0.5 i\left(A_{2}-A_{1}\right) & >2 \alpha_{1}, \\
\alpha_{2}+12.93 \alpha_{1} & >13.93 A_{1}, \\
\alpha_{2}+12.93 \alpha_{1}+6.97 i\left(A_{2}-A_{1}\right) & >3.48 A_{2} .
\end{aligned}
$$

Two additional analogous conditions define the maximum number of cells that the lattice made of these materials can contain:

$$
\begin{aligned}
& n<\frac{2 \alpha_{2}+0.5 A_{2}-4 \alpha_{1}}{A_{2}-A_{1}}, \\
& n<\frac{0.5 A_{2}+0.14 \alpha_{2}+1.86 \alpha_{1}-A_{1}}{A_{2}-A_{1}} .
\end{aligned}
$$

If we need to design a lattice of more complex shape or just a lattice without the vertical line of symmetry or consisting, for example, of an odd number of cells, the formulae for the cells' CTEs and the lattice-material selection can be obtained in similar way. If two substrates are connected by a one-row lattice with three cells or more, they also can be connected by a lattice containing two or more rows. This may be advantageous if a lattice possessing a particular ratio of width to height is preferable.

\section{General algorithm of lattice design}

Assembling all the reasoning presented in the previous sections, this algorithm is effective for latticetailoring:

(1) Choose the initial number of cells in the lattice.

(2) Using the formulae (14), (16), or (18), find the CTEs $\alpha_{i j}$ in all cells of the lattice as functions of the substrate CTEs $A_{1}$ and $A_{2}$.

(3) Choose the materials of the lattice accounting for the relations (15), (17), (19), or (20), and find the ratio $\alpha_{2} / \alpha_{1}$.

(4) Check the existence of solutions to equations (6) at calculated values of normalized CTEs of the current cell $\overline{\alpha_{i}} / \alpha_{1}=\alpha_{i j} / \alpha_{1}, i=1,2,3$, and the chosen ratio of $\alpha_{2} / \alpha_{1}$.

(5) If the solution exists, find the skew angles of the current cell. Then repeat the previous step with the next cell of the lattice. 
(6) If the solution does not exist, there are three options:

(a) Choose lattice materials with a higher value of the ratio $\alpha_{2} / \alpha_{1}$.

(b) Use unequal negative skew angles adjacent to the cell members in order to provide a wider range of cell CTEs.

(c) Reduce the number of cells in the lattice.

(7) After Step (6), repeat Step (4).

(8) The lattice design halts when this procedure is performed for all cells in the lattice. If the lattice can be successfully designed, it may be possible to increase the number of units cells and redesign the lattice, beginning at Step (1).

The initial number of lattice cells is determined heuristically, accounting for the geometry of the substrates and the difference between their CTEs. The larger the difference, the fewer cells the lattice can contain. Problem 6 from the next section will illustrate this. The final number of cells is determined through Step (6)(c) in the design algorithm.

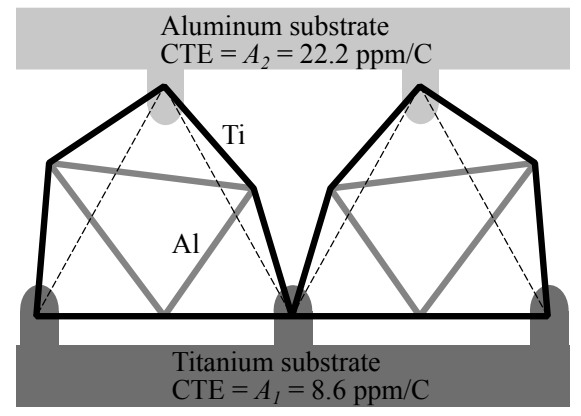

Figure 8. Problem 1: Two-cell aluminum-titanium lattice to connect aluminum and titanium substrates.

\section{Examples}

Problem 1. This is an example of connecting titanium and aluminum substrates by a two-cell lattice made also of titanium and aluminum. So $\alpha_{1}=A_{1}=8.6 \mathrm{ppm} / \mathrm{C}, \alpha_{2}=A_{2}=22.2 \mathrm{ppm} / \mathrm{C}$, and $\alpha_{2} / \alpha_{1}=2.58$. For these values of $\alpha_{1}$ and $\alpha_{2}, \Omega=[-0.581,1.1135]$. From (14), we find $\alpha_{11} / \alpha_{1}=\alpha_{21} / \alpha_{1}=A_{1} / \alpha_{1}=1$, $\alpha_{12} / \alpha_{1}=\alpha_{23} / \alpha_{1}=0.25 A_{2} / \alpha_{1}=0.64535$, and $\alpha_{13} / \alpha_{1}=\alpha_{23} / \alpha_{1}=0.5 A_{1} / \alpha_{1}-0.25 A_{2}=-0.14535$. All values of $\alpha_{i j}$ belong to $\Omega$. Now using formulae (11), the skew angles in the left cell can be calculated as $t_{1}=0.0^{\circ}, t_{2}=12.9^{\circ}$, and $t_{3}=27.1^{\circ}$ (Figure 8 ).

In the problem of the two-cell lattice connecting titanium and aluminum substrates, the inequalities (15) define a region of allowable values of $\alpha_{1}$ and $\alpha_{2}$; the region is plotted in Figure 9. For this case, the third and fourth inequalities in (15) are the strongest. Their intersection provides a minimum of $\alpha_{2}=17.14 \mathrm{ppm} / \mathrm{C}$ with corresponding $\alpha_{1}=7.94 \mathrm{ppm} / \mathrm{C}$.

The utility of the lattice adapter can be illustrated by this example. For comparison, a bimetallic strip consisting of titanium and aluminum layers of the same thickness welded together and uniformly heated to $100^{\circ} \mathrm{C}$ will be bent due to thermal-expansion mismatch [Timoshenko 1925]. The maximum stress during heating of this bimetallic strip [Timoshenko 1925] is $70.5 \mathrm{MPa}$. A pinned lattice adapter experiences no thermal distortion or thermal stress. 


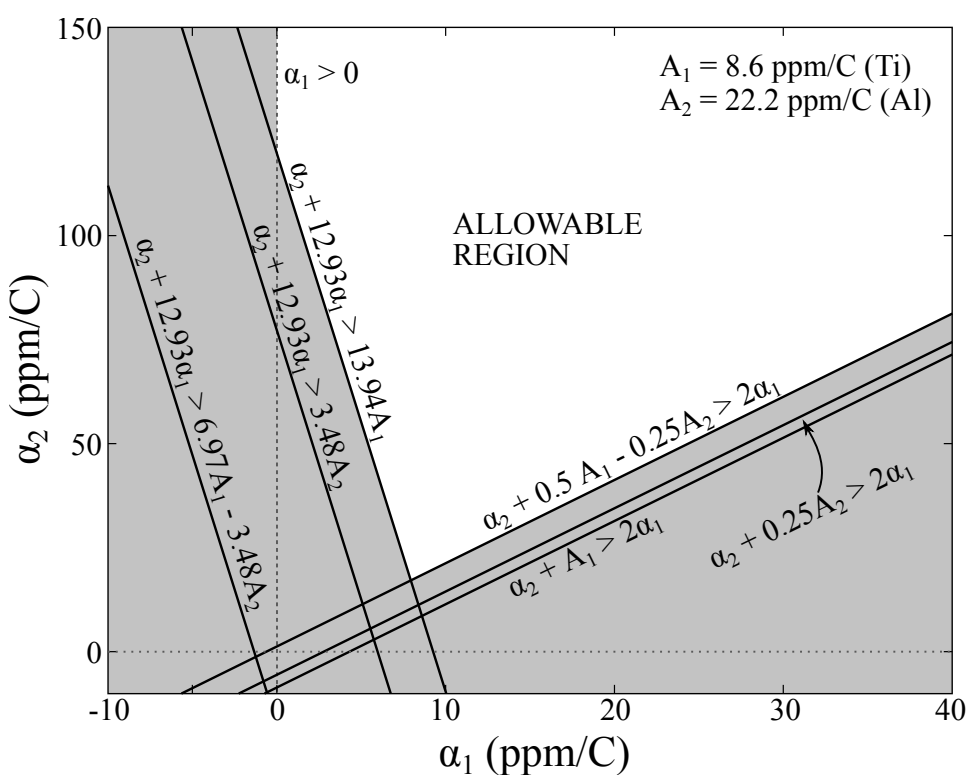

Figure 9. Problem 1: The white region indicates the ranges for the CTEs of the lattice materials that can be used to design the lattice for Problem 1. The lines defining the allowable region are the inequalities in (15). Note the difference in the scales of the two axes; for small or moderately large values of $\alpha_{1}$, large values of $\alpha_{2}$ are implied.

Problem 2. The same lattice materials can be used to connect other substrates that have CTEs that are more widely different, for example, zirconium and lead. In this case, $A_{1}=5.7 \mathrm{ppm} / \mathrm{C}$ and $A_{2}=28 \mathrm{ppm} / \mathrm{C}$ with $\Omega=[-0.581,1.1135]$. Then $\alpha_{11} / \alpha_{1}=\alpha_{21} / \alpha_{1}=A_{1} / \alpha_{1}=0.66279, \alpha_{12} / \alpha_{1}=\alpha_{23} / \alpha_{1}=0.25 A_{2} / \alpha_{1}=$ 0.81395 , and $\alpha_{13} / \alpha_{1}=\alpha_{23} / \alpha_{1}=0.5 A_{1} / \alpha_{1}-0.25 A_{2}=-0.48256$. Again, $\alpha_{i j}$ belong to $\Omega$. The skew angles for the left cell are $t_{1}=12.71^{\circ}, t_{2}=8.65^{\circ}$, and $t_{3}=30.48^{\circ}$ (Figure 10). It is seen that skew angles of the lattice cells are greater than those from Problem 1. We have allowed $t_{3}>30^{\circ}$ because these are the exterior sides of the lattice, and hence, there are no adjacent cells to cause interference. It does however make impossible the addition of further cells.

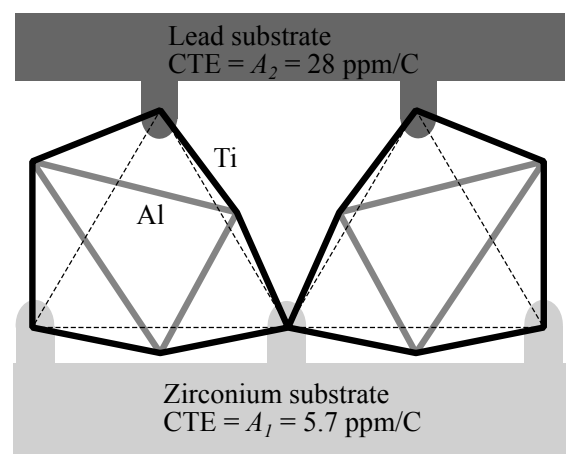

Figure 10. Problem 2: Two-cell aluminum-titanium lattice to connect zirconium and lead substrates. 


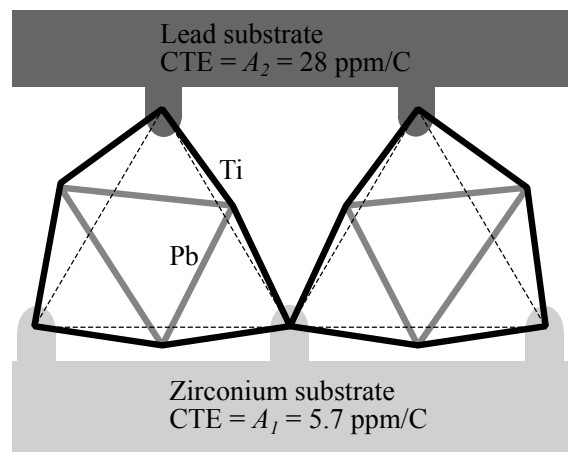

Figure 11. Problem 3: Two-cell lead-titanium lattice to connect zirconium and lead substrates.

Problem 3. We can connect the same substrates as in Problem 2 using other materials for the lattice, for example, titanium and lead. While these materials are unlikely candidates for lattice construction, their widely differing CTEs make them good example materials. In this case, $\alpha_{1}=8.6 \mathrm{ppm} / \mathrm{C}$, $\alpha_{2}=28.0 \mathrm{ppm} / \mathrm{C}$, and $\alpha_{2} / \alpha_{1}=3.25 . A_{1}$ and $A_{2}$ and therefore the values of $\alpha_{i j}$ have the same values as in Problem 2. For this combination of lattice materials, $\Omega=[-1.256,1.162]$. All values of $\alpha_{i j}$ belong to $\Omega$. From formulae (11), the skew angles in the left cell can be calculated as $t_{1}=10.0^{\circ}, t_{2}=6.5^{\circ}$, and $t_{3}=25.7^{\circ}$ (Figure 11). In this lattice, the ratio $\alpha_{2} / \alpha_{1}$ is greater than in the lattices of the previous problems, which is why the lattice cells are less skewed.

Problem 4. In this problem, we design a three-cell aluminum-titanium lattice connecting aluminum and titanium substrates. It is possible because the ratio of CTEs of the substrates satisfies $2.5<\alpha_{2} / \alpha_{1}<2.7515$. Using formulae (16), we find $\alpha_{11} / \alpha_{1}=\alpha_{21} / \alpha_{1}=\alpha_{31} / \alpha_{1}=1, \alpha_{12} / \alpha_{1}=\alpha_{33} / \alpha_{1}=1.04, \alpha_{13} / \alpha_{1}=\alpha_{32} / \alpha_{1}=$ -0.54 , and $\alpha_{22} / \alpha_{1}=\alpha_{23} / \alpha_{1}=0.25$. The skew angles for the first cell on the left are $t_{1}=0.0^{\circ}, t_{2}=-3.0^{\circ}$, and $t_{3}=32.6^{\circ}$. The skew angles for the second cell are $t_{1}=0.0^{\circ}, t_{2}=21.0^{\circ}$, and $t_{3}=21.0^{\circ}$ (Figure 12). Here, although $\alpha_{2} / \alpha_{1} \in \Omega$, this ratio is very close to the minimum boundary of $\Omega$. That is why $t_{3}$ in the first cell on the left and $t_{2}$ in last cell on the right are greater than $30^{\circ}$. In this case, it is admissible because these skew angles do not cause overlapping with adjoining lattice cells but force a limit to the number of cells in this lattice to be three.

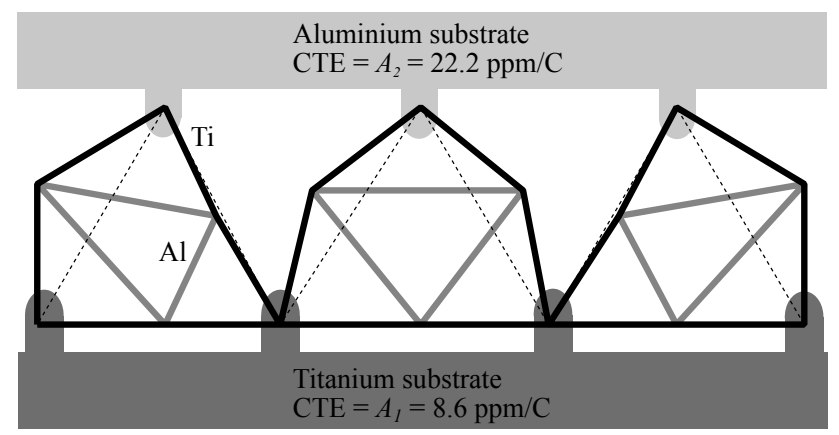

Figure 12. Problem 4: Three-cell titanium-aluminum lattice to connect titanium and aluminum substrates. 


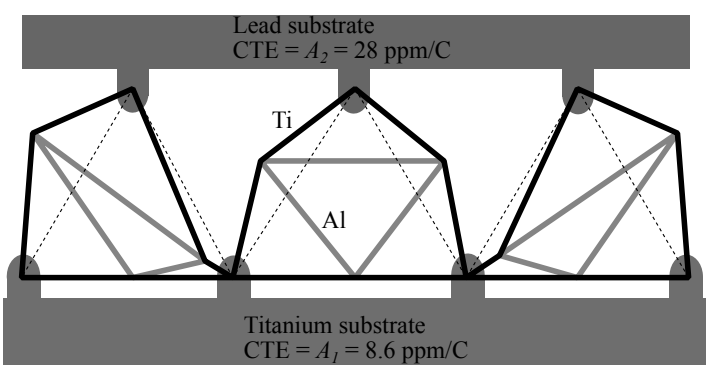

Figure 13. Problem 5: Three-cell titanium-aluminum lattice to connect titanium and lead substrates. The available range of thermal expansion is expanded by allowing the six skew angles to be independent.

Problem 5. Suppose there is a need to connect titanium and lead substrates by a three-cell titaniumaluminum lattice. From formulae (16), we have $\alpha_{11} / \alpha_{1}=\alpha_{21} / \alpha_{1}=\alpha_{31} / \alpha_{1}=1, \alpha_{12} / \alpha_{1}=\alpha_{33} / \alpha_{1}=$ 1.375, $\alpha_{13} / \alpha_{1}=\alpha_{32} / \alpha_{1}=-0.875$, and $\alpha_{22} / \alpha_{1}=\alpha_{23} / \alpha_{1}=0.25$. For the chosen lattice materials, $\Omega=[-0.581,1.1135]$. As we can see, $\alpha_{12} / \alpha_{1}=\alpha_{33} / \alpha_{1} \notin \Omega$ and $\alpha_{13} / \alpha_{1}=\alpha_{32} / \alpha_{1} \notin \Omega$. To overcome this for $\alpha_{12} / \alpha_{1}$, we can use negative nonsymmetric skew angles $\theta_{3} \neq \theta_{4}$, and for $\alpha_{13} / \alpha_{1}$, we can use symmetric angles greater than $30^{\circ}$; this will not lead to the overlapping of the cells because these skew angles are adjacent to the external sides of the lattice. The skew angles for the second cell are the same as in Problem 4. The solution for the first cell on the left is not unique; for example, the skew angles that satisfy (6) may be $\theta_{1}=\theta_{2}=0.0^{\circ}, \theta_{3}=-30.0^{\circ}, \theta_{4}=-6.2^{\circ}$, and $\theta_{5}=\theta_{6}=35.3^{\circ}$. Figure 13 is a sketch of the lattice.

Problem 6. If the substrate materials have CTEs that are relatively similar $\left(A_{2} / A_{1} \lesssim 2\right)$, we can design a lattice consisting of four cells and more. For example, suppose we would like to connect titanium $\left(A_{1}=8.6 \mathrm{ppm} / \mathrm{C}\right)$ and stainless-steel $\left(A_{2}=17.3 \mathrm{ppm} / \mathrm{C}\right)$ substrates with an aluminum-titanium lattice. For such materials, the maximum total number of cells in the lattice according to inequalities (20) is 4 . Using formulae (18), we have $\alpha_{11} / \alpha_{1}=\alpha_{21} / \alpha_{1}=1, \alpha_{12} / \alpha_{1}=0.5 A_{1} / \alpha_{1}-0.25 A_{2} / \alpha_{1}=0.03, \alpha_{13} / \alpha_{1}=$ $0.25 A_{2} / \alpha_{1}=0.5, \alpha_{22} / \alpha_{1}=A_{1} / \alpha_{1}-0.75 A_{2} / \alpha_{1}=-0.5$, and $\alpha_{23} / \alpha_{1}=0.75 A_{2} / \alpha_{1}-0.5 A_{1} / \alpha_{1}=1.0$. Now, using (11), for Cell $1, t_{1}=0.0^{\circ}, t_{2}=24.6^{\circ}$, and $t_{3}=16.1^{\circ}$. For Cell $2, t_{1}=0.0^{\circ}, t_{2}=32.0^{\circ}$, and $t_{3}=0.0^{\circ}$ (Figure 14). Two other cells are symmetric with respect to the vertical line passing through the middle of the lattice. Note that, if the second substrate is made from ferritic stainless steel with CTE $A_{2}=9.9 \mathrm{ppm} / \mathrm{C}$, then the maximum number of the cells in the lattice would be 22 .

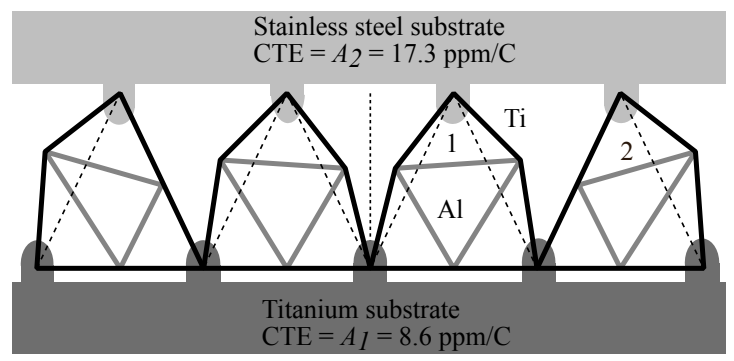

Figure 14. Problem 6: Four-cell aluminum-titanium lattice for stainless steel and titanium substrates. 
The solutions to the problems considered above show that, the more the substrates' CTEs differ, the more the lattice cells are skewed. On the other hand, the higher the ratio of CTEs of the lattice materials, the less the cells of the lattice are skewed. Also, different skew angles adjacent to the same side of the cell can provide a wider range of CTEs compared to corresponding skew angles equal to each other.

\section{Conclusion}

The anisotropic planar lattices described in this paper provide a structural option for connecting materials with differing CTEs without generating thermal stresses during temperature excursions. Each cell is composed of internal and external triangles made of materials with different CTEs and pin-connected at three locations. The internal triangle is regular whereas the external triangle is deformed with varying skew angles. As a consequence of the variation in skew angles in a single cell, the cells have anisotropic CTEs. Combining cells with anisotropic CTEs into a pin-jointed lattice provides the capability to achieve desirable, and differing, CTEs on the bottom and top edges of the lattice. Such lattices can therefore be used as transition elements between two parts of a structure (substrates) with different CTEs, and as a result, the whole structure will be free of thermal stresses. Additionally, these lattices are relatively stiff; the isotropic variants are nearly optimally stiff for a structure of this nature [Steeves et al. 2007]. Alternative options for adapters for thermal mismatch mitigation either induce thermal stresses and curvatures or are very compliant.

The design strategy described herein provides a systematic process for choosing the geometric configuration of a single-row lattice that connects substrates of known materials. In particular, guidance on the choice of the materials that would be appropriate to connect the substrates is given, based upon the CTEs of the substrate materials. The design process for single rows of lattice can be extended to multiple rows if that provides a preferable aspect ratio for the adapter. A key limitation to this lattice system is that there are stringent limits on the maximum number of cells that can be used. As the difference between the substrate CTEs increases, the maximum number of lattice cells is reduced because the total deflections that must be accommodated increase with lattice length. An option for mitigating this limitation is to use multirow lattices and permit rotation of the lattice cells. Such topics are the subject of ongoing research.

\section{References}

[Berger et al. 2011] J. Berger, C. Mercer, R. M. McMeeking, and A. G. Evans, "The design of bounded biomaterial lattices that combine low thermal expansion with high stiffness”, J. Am. Ceram. Soc. 94:S1 (2011), S42-S54.

[Dang 2008] C. H. Dang, "Coefficient of thermal expansion adaptor”, US Patent No. 2008/0160274 A1, 2008, Available at http://www.google.com/patents/US20080160274.

[Gdoutos et al. 2013] E. Gdoutos, A. A. Shapiro, and C. Daraio, "Thin and thermally stable periodic metastructures", Exp. Mech. 53:9 (2013), 1735-1742.

[Gibiansky and Torquato 1997] L. V. Gibiansky and S. Torquato, "Thermal expansion of isotropic multiphase composites and polycrystals", J. Mech. Phys. Solids 45:7 (1997), 1223-1252.

[Jefferson et al. 2009] G. Jefferson, T. A. Parthasarathy, and R. Kerans, "Tailorable thermal expansion hybrid structures", Int. J. Solids Struct. 46:11-12 (2009), 2372-2387.

[Lakes 1996] R. S. Lakes, "Cellular solid structures with unbounded thermal expansion”, J. Mater. Sci. Lett. 15:6 (1996), 475-477.

[Lehman and Lakes 2013] J. Lehman and R. S. Lakes, "Stiff lattices with zero thermal expansion and enhanced stiffness via rib cross section optimization”, Int. J. Mech. Mater. Des. 9:3 (2013), 213-225. 
[Sigmund and Torquato 1996] O. Sigmund and S. Torquato, "Composites with extremal thermal expansion coefficients", Appl. Phys. Lett. 69:21 (1996), 3203-3205.

[Sigmund and Torquato 1997] O. Sigmund and S. Torquato, "Design of materials with extreme thermal expansion using a three-phase topology optimization method", J. Mech. Phys. Solids 45:6 (1997), 1037-1067.

[Steeves et al. 2007] C. A. Steeves, S. L. dos Santos e Lucato, M. He, E. Antinucci, J. W. Hutchinson, and A. G. Evans, "Concepts for structurally robust materials that combine low thermal expansion with high stiffness", J. Mech. Phys. Solids 55:9 (2007), 1803-1822.

[Timoshenko 1925] S. P. Timoshenko, "Analysis of bi-metal thermostats", J. Opt. Soc. Am. 11:3 (1925), 233-255.

[Yousefiani et al. 2009a] A. Yousefiani, J. M. Comfort, J. G. Vollmer, and M. L. Hand, "Joined composite structures with a graded coefficient of thermal expansion for extreme environment applications", US Patent No. 2009/0266870 A1, 2009, Available at http://www.google.com/patents/US20090266870.

[Yousefiani et al. 2009b] A. Yousefiani, J. G. Vollmer, M. L. Hand, and J. M. Comfort, "Built-up composite structures with a graded coefficient of thermal expansion for extreme environment applications", US Patent No. 2009/0269497 A1, 2009, Available at http://www.google.com/patents/US20090269497.

Received 13 Dec 2013. Revised 7 Feb 2014. Accepted 25 Mar 2014.

MARINA M. TOROPOVA: toropova@utias .utoronto.ca

Institute for Aerospace Studies, University of Toronto, 4925 Dufferin Street, Toronto, ON M4H 5T6, Canada

Craig A. SteEves: csteeves@utias utoronto.ca

Institute for Aerospace Studies, University of Toronto, 4925 Dufferin Street, Toronto, ON M4H 5T6, Canada 


\title{
JOURNAL OF MECHANICS OF MATERIALS AND STRUCTURES
}

\author{
msp.org/jomms
}

\author{
Founded by Charles R. Steele and Marie-Louise Steele
}

EDITORIAL BOARD

ADAIR R. AGUIAR

KATIA BERTOLDI

University of São Paulo at São Carlos, Brazil

DAVIDE BIGONI

Harvard University, USA

IWONA JASIUK

University of Trento, Italy

Thomas J. PENCE

University of Illinois at Urbana-Champaign, USA

YASUHIDE SHINDO

Michigan State University, USA

DAVID STEIGMANN

Tohoku University, Japan

University of California at Berkeley

\section{ADVISORY BOARD}

J. P. CARTER University of Sydney, Australia

R. M. Christensen Stanford University, USA

G. M. L. GLADWELL University of Waterloo, Canada

D. H. Hodges Georgia Institute of Technology, USA

J. HUTCHINSON Harvard University, USA

C. HwU National Cheng Kung University, Taiwan

B. L. KARIHALOO University of Wales, UK

Y. Y. KIM Seoul National University, Republic of Korea

Z. Mroz Academy of Science, Poland

D. PAMPlonA Universidade Católica do Rio de Janeiro, Brazil

M. B. RubiN Technion, Haifa, Israel

A. N. SHUPIKOV Ukrainian Academy of Sciences, Ukraine

T. TARNAI University Budapest, Hungary

F. Y. M. WAN University of California, Irvine, USA

P. WRIGGERS Universität Hannover, Germany

W. YANG Tsinghua University, China

F. ZIEGLER Technische Universität Wien, Austria

PRODUCTION production@msp.org

SILVIO LEVY Scientific Editor

Cover photo: Mando Gomez, www.mandolux.com

See msp.org/jomms for submission guidelines.

JoMMS (ISSN 1559-3959) at Mathematical Sciences Publishers, 798 Evans Hall \#6840, c/o University of California, Berkeley, CA 94720-3840, is published in 10 issues a year. The subscription price for 2014 is US \$555/year for the electronic version, and $\$ 710 /$ year (+\$60, if shipping outside the US) for print and electronic. Subscriptions, requests for back issues, and changes of address should be sent to MSP.

JoMMS peer-review and production is managed by EditFLow ${ }^{\circledR}$ from Mathematical Sciences Publishers.

PUBLISHED BY

mathematical sciences publishers

nonprofit scientific publishing

http://msp.org/

(C) 2014 Mathematical Sciences Publishers 


\title{
Journal of Mechanics of Materials and Structures
}

\author{
Volume 9, No. $2 \quad$ March 2014
}

Plane waves at the boundary of two micropolar thermoelastic solids with distinct conductive and thermodynamic temperatures

Rajneesh Kumar, Mandeep Kaur and Satish C. RaJvanshi

Dynamic compression of square tube cellular structures RYAN L. HOLLOMAN, KaRTHIKEyan Kandan, VikRam DeshPande and HaYdn N. G. WadLey

Dynamic response of twin lined shells due to incident seismic waves

J. P. DWIVEdi, V. P. Singh and RADHA KRISHNA LAL 183

Solutions of the von Kármán plate equations by a Galerkin method, without inverting the tangent stiffiness matrix.

Honghua Dai, Xiaokuí Yue and Satya N.Atluri 195

Bimaterial lattices with anisotropic thermal expansion

Marina M. Toropova and Craig A. Steeves

Origin and effect of nonlocality in a composite STEWART A. Silling 